\title{
Efficient Use of Cloud Computing in Medical Science
}

\author{
Avula Tejaswi, Nela Manoj Kumar, Gudapati Radhika, Sreenivas Velagapudi \\ Department of Computer Science and Engineering, K L University, Guntur, India \\ Email: avula.tejaswi9@gmail.com,manojk0531@gmail.com,radhika.gudapati@gmail.com \\ velagapudisreenivas@gmail.com
}

Received April 23, 2012; revised July 8, 2012; accepted July 16, 2012

\begin{abstract}
Technology has grown rapidly with scientific advancement over the world in recent decades. Therefore, there is a need to redesign the medical system to meet their better needs. With the advent of cloud computing the doctors can keep their information about their critical diseases, critical cases and sophisticated problem they phased .cloud computing has made it possible to solve many complex problems very fast and at a lower cost in less time. Almost all doctors in various disciplines uses cloud computing to solve their problems. In this paper, attention is given to possible implementation of Cloud computing technology in the medical field, especially in hospitals where there is intensive need of computers for better cure for the diseases.
\end{abstract}

Keywords: Cloud; Diseases; Treatment; Medical; Information; Internet

\section{Introduction}

Computers play a prominent role in the educational sector, as well as in business and industry sectors. They can also play major role in medical field. With availability of high-end information rarely matches with doctor need in a typical solution. In medical field a doctor can keep information on cloud to share among the world. A doctor can find a new complex disease in a patient; he/she will get the symptoms of the diseases, how to cure the disease and all the information about the disease. The doctor can place the information on the cloud. If the same disease came to another patient in world that doctor can get information about the disease and doctor can cure the disease for the patient. With the help of cloud computing the doctor in different countries can get information about complex diseases. The information in cloud will be encrypted [1] for the security purpose.

\section{Cloud Computing}

Cloud technology [2] provides computation, software, data access, and storage services that do not require end-user knowledge of the physical location and configuration of the system that delivers the services. Cloud technology providers deliver applications via the internet, which are accessed from a web browser, while the business software and data are stored on servers at a remote location. Cloud computing is highly scalable and creates virtualized resources that can be made available to users. Users do not require any special knowledge about the concept of Cloud computing to connect their computers to the server where applications have been installed and use them. Users can communicate through Internet with remote servers. These servers can exchange their computing slots themselves. Cloud computing is one of the new technologies [3] likely to have a significant impact on the teaching and learning environment.

\section{Types of Cloud Services}

In cloud technology the information is shared from clients to the organization through the virtual data centers. This virtual data centers has all the required information (Figure 1). The cloud technology model includes [1]:

1) SaaS (Software as a service);

2) PaaS (Platform as a service);

3) IaaS (Infrastructure as a service).

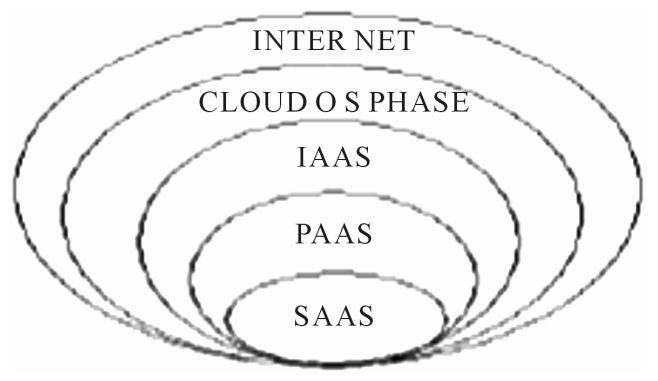

Figure 1. Services of cloud. 


\subsection{SaaS (Software as a Service)}

Saas (Figure 1) is simply the cloud vender providing the given piece of software you want to use on their servers. It is a way of delivering applications over the Internet-as a service. Instead of installing and maintaining software, you simply access it via the Internet, freeing yourself from complex software and hardware management. SaaS [4] applications are sometimes called Web-based software, ondemand software, or hosted software. Whatever the name, SaaS applications run on a SaaS provider's servers.

\subsection{PaaS (Platform as a Service)}

To follows the heels of SaaS, platform as a service (PaaS) [2] is another application delivery model. PaaS supplies all the resources required to build applications and services completely from the internet without having to download or install software. PaaS services include application design development, testing, deployment, and hosting. It provides infrastructure on which software developers can build new applications or extend existing ones without the cost and complexity of buying and managing the underlying hardware and software and provisioning hosting capabilities. PaaS generally offers some support to help the creation of user interfaces, and is normally based on HTML or JavaScript.

\subsection{IaaS (Interface as a Service)}

This offers remote delivery of an entire computer infrastructure. Managed hosting and development environments are the services included in IaaS (Figure 1). The user can buy the infrastructure according to the requirements at any particular point of time instead of buying the infrastructure that might not be used for months. IaaS operates on a "Pay as you go" model ensuring that the users pay for only what they are using.

\section{Types of Cloud Computing}

\subsection{Public Cloud}

Public cloud (Figure 2) or external cloud describes cloud computing in the traditional mainstream sense, where by resources are dynamically provisioned on a fine-grained, self-service basis over the internet via web applications/web services, from an off-site third-party provider who shares resources and bills on a fine-grained utility computing basis. A public cloud is one based on the standard cloud computing model, in which a service provider makes resources, such as applications and storage, available to the general public over the Internet. Public cloud services may be free or offered on a pay-per-usage model.

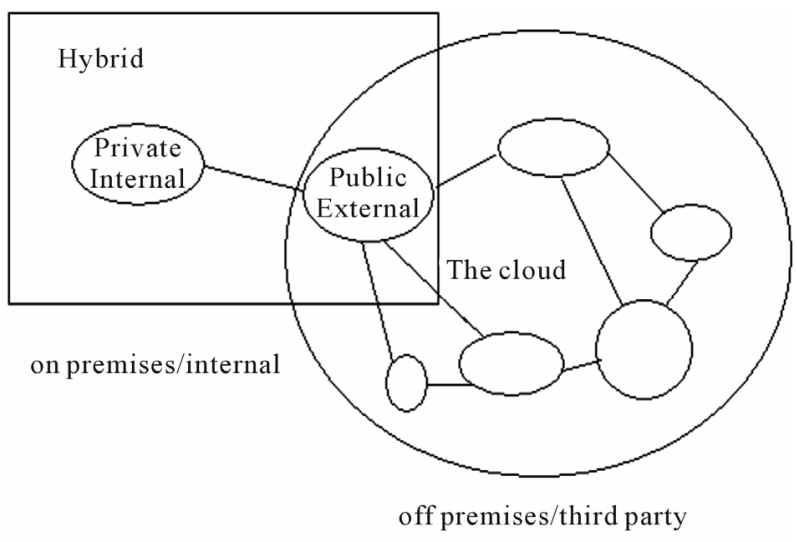

Cloud Computing Types

Figure 2. Types of cloud computing.

\subsection{Private Cloud}

Private cloud (Figure 2) (also called internal cloud or corporative cloud) is used in within the organization or corporation. It provides hosted services to very limited number of people behind a firewall. Advances in virtualization and distributed computing have allowed corporate network and datacenter administrators to effectively become service providers that meet the needs for their "customers" within the corporation.

\subsection{Hybrid Cloud}

It is a cloud computing environment in which an organization provides and manages some resources in-house and has others provided externally. For example an organization might use a public cloud service, such as Amazon's Elastic Cloud (EC2) for general computing but store customer data within its own data center. Although cloud computing is often said to be the future of the industry, the hybrid cloud (Figure 2) model is more prevalent for a number of reasons. Large enterprises often already have substantial investments in the infrastructure required to provide resources in-house. Furthermore, many organizations would prefer to keep sensitive data under their own control to ensure security [1].

\section{Methodology}

Now a day's the people in the world are sick with so many health problems and there are some problems which are unknown to the doctors also so in that situations the doctors need to know how to treat that patients, to overcome this type of problems we suggested a new era of technology called cloud computing in medical sciences. Here the doctors has to prepare some document whenever he got any critical cases which he has ever seen and treated in that situation he has to make it a document that is how to treat that patient. There are some 
points that a doctor need to mention while documenting regarding a problem they are

1) Symptoms;

2) Analysis;

3) Treatment.

\subsection{Symptoms}

Here if the doctor found any disease that he has ever seen then, at first he has to mention the symptoms of that disease and make a note of it to inform other doctors, if any doctor faces the same disease cases. It is possible only if the other doctor is friend to him or he is a doctor in another hospital in the same city/region.

\subsection{Analysis}

After verifying all symptoms of the patient the doctor conclude that what type of disease it is and how it came and is it cures for normal treatment or he has to take a special treatment for that disease all these concerns are analyzed.

\subsection{Treatment}

After analyzing all the things regarding the disease the doctor will start the treatment, here in this phase doctor will give medicines to the patient and he has to mention what the medicines are given to the patient and the dosage of it. There are some points that the doctor has to follow while treating the patient they are. Mention the medicines and dosage which are given to the patient. Observe the patient while the treatment is going on whether is there any changes in his body or health. Mention which type of treatment he has given to the patient. Guide the patient, the doctor has to mention all the above while treating the patient.

\section{Implementation}

\subsection{Existing System}

In the Existing system the doctors didn't use any cloud to inform about new diseases in patients so it is very difficult to cure and they don't know how to save people in the world from that disease because, if the disease found in china then the doctor in India may not know about that and they have to know only through media even though many people were died of that disease so the death rate is increasing enormously.

\subsection{Proposed System}

Here in this proposed system we are going to introduce cloud computing in medical sciences because it is very easiest way to know all the doctors over the world through the cloud about the diseases which are very recently found and how to cure it, by applying this we can reduce death rate. By Sharing the information on cloud the reputation of hospital and doctor will increase globally.

The doctors will give treatment for all the diseases regularly in general but there are some cases which are very difficult cure in such cases the doctor willing to cure that as soon as possible and at last he will cure it, in the same way if any doctor faces the same problem in the

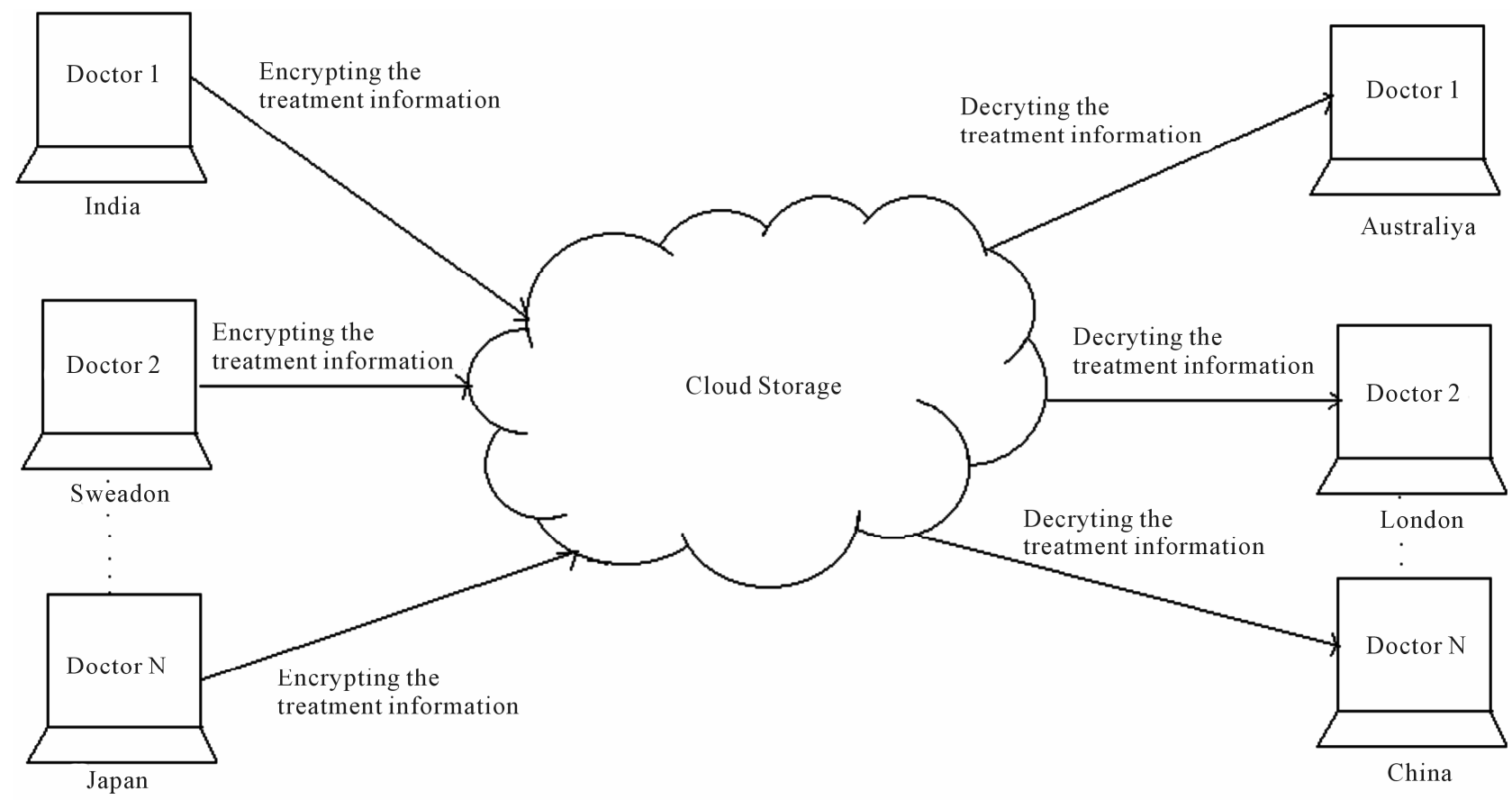

Figure 3. Deploying medical information to the cloud and retrieving information from cloud. 
patient then it is very easy to inform to that doctor about the disease and how to cure it if the second doctor is friend of another doctor or both the doctors are in the same city, if the same problem is arises in another country then it is very difficult to inform that doctor about the treatment and how to cure it.

So we are implementing this cloud (Figure 3) in medical sciences, here every doctor who is facing rare cases need to prepare a document about symptoms, analysis of the disease and how to cure it and what are the medicines he has given to cure it, all these information will documented by the doctor and he has to keep it in the cloud.

Let us take example disease like swine-flu, swine flu is an one kind of fever and the first case of swine flu is found in china at that time nobody knows about that and then they found symptoms and medicines for that and how to treat. After some time we found those swine-flu cases in all over the world and some people were died of this swine-flu. If the doctors made any document and keep it in cloud whenever they found at first, then it is very easy to cure this type of cases all over the world.

\section{Conclusion}

In this paper we introduced a cloud computing technology in medical science. It is widely used for doctors and this technology introduces an easy way to curing the pa- tients, if any new disease is occurred. Here, we utilize the existing enhancement of cloud security protocols for authenticating the treatment information. By using cloud computing will reduce the time required for patient to become into normal stage. Cloud computing will create a major impact on medicine, and contribute to an overall improvement in its quality. To conclude that if the encrypted treatment information of the patients is uploaded to the cloud, only authenticated receivers (doctors) can decrypt it there by we can enhance the security to the information.

\section{REFERENCES}

[1] V. Sreenivas and C. Narasimham, "Enhancing the Security for Information with Virtual Data Centers in Cloud," Future Wireless Networks and Information Systems, Vol. 143, 2012, pp. 277-282.

[2] B. Hayes, "Cloud Computing," Communications of the ACM, Vol. 51, No. 7, 2008, pp. 9-11. doi:10.1145/1364782.1364786

[3] M. Pokharel, Y.-H. Yoon and J. S. Park, "Cloud Computing in System Architecture,” International Symposium on Computer Network and Multimedia Technology, Wuhan, 18-20 January 2009, pp. 1-5.

[4] V. Sreenivas and C. Narasimham "A Cloud Model to Implement SaaS,” Advanced Materials Research, Vol. 341-342. 2011, pp. 499-503. 